\title{
Advanced translation and cultural adaption of the LYMPH-Q Upper Extremity Module from English to Danish
}

\author{
Christoffer Bing Madsen ${ }^{1,2}\left(\right.$ C $\cdot$ Lotte Poulsen ${ }^{1,2}\left(\right.$ Mads Gustaf Jørgensen $^{1,2}\left(\mathbb{D}^{\circ} \cdot\right.$ Mike Mikkelsen Lorenzen $^{1,2}$. \\ Elena Tsangaris $^{3}$ - Anne Klassen ${ }^{4}$ (i) . Jens Ahm Sørensen ${ }^{1,2}$ (I)
}

Received: 1 September 2021 / Accepted: 13 September 2021 / Published online: 12 October 2021

(c) The Author(s) 2021

\begin{abstract}
Background To better quantify the impact that breast cancer-related arm lymphedema (BCRL) has on health-related quality of life (HR-QOL), a disease-specific patient-reported outcome measure (PROM) is needed. The LYMPH-Q Upper Extremity Module was recently developed for patients with BCRL. The aim of this study was to perform an advanced translation and culturally adapt the LYMPH-Q Upper Extremity Module for use in Denmark.

Methods The LYMPH-Q Upper Extremity Module was translated into Danish according to the guidelines of the International Society for Pharmacoeconomics and Outcomes Research (ISPOR) and the World Health Organization (WHO). The process included two forward and one back translation, an expert panel meeting, and cognitive debriefing interviews with patients. The focus of the translation was to develop a Danish version that used appropriate patient-friendly language while maintaining the meaning of the items, instructions and response options.

Results The two forward translations resulted in minor differences in terminology. These discrepancies were discussed among the translators and a harmonized Danish version 1 was achieved. Comparison of the back translation to the original English version identified 14 items/instructions/response options that required re-translation. Subsequently, experts helped to identify and resolve the language for 10 items/instructions/response options that did not maintain the same meaning as the English version. Participants in the cognitive debriefing interviews did not report any difficulties with understanding the items/instructions/response options.
\end{abstract}

Conclusions The translation and cultural adaption process led to the development of a conceptually equivalent Danish version of the LYMPH-Q Upper Extremity Module.

Level of Evidence: Not gradable

Keywords Patient-reported outcome measure $\cdot$ PROM $\cdot$ Health-related quality of life $\cdot$ HRQoL $\cdot$ Breast cancer . Lymphedema $\cdot$ Translation and cultural adaption

\section{Introduction}

Christoffer Bing Madsen

christoffer.bing.madsen@rsyd.dk

1 Research Unit for Plastic Surgery, Odense University Hospital, Odense, Denmark

2 University of Southern Denmark, Odense, Denmark

3 Patient-Reported Outcomes, Value \& Experience (PROVE) Center, Brigham and Women's Hospital, Harvard Medical School, Boston, MA, USA

4 Department of Pediatrics, McMaster University, Hamilton, ON, Canada
Breast cancer is the second most common cancer in women in Denmark. Between 2012 and 2016, over 4500 new cases were reported annually, and by 2016 , approximately 66,372 Danish women were living with a breast cancer [1].

Lymphedema of the arm is a well-known side effect of breast cancer treatment, and is characterized by edema of the arm, hand, and fingers resulting in inflammation and fibrosis [2]. According to DiSipio et al. [3], close to $20 \%$ of all breast cancer cases develop unilateral arm lymphedema [3]. A diagnosis of breast cancer-related lymphedema (BCRL) may result in changes to the appearance of the arm, increased 
pain and risk of infection, and decreased arm function such as range of motion $[4,5]$.

Objective assessments such as arm circumference, water displacement, and bioimpedance have traditionally been used to assess outcomes of lymphedema treatment [6]. However, studies have shown a discordance between objective measurements and symptoms (e.g., pain, the arm feels heavy, and the arm feels numb) experienced by patients [7]. For instance, Sackey et al. [8] found that patients without objective lymphedema, but who are experiencing symptoms, are more likely to experience a diminished overall health-related quality of life (HR-QOL) in the long-term [8]. Furthermore, Jørgensen et al. found that patients with BCRL had significantly lower HR-QOL than patients without BCRL in 16 out of 18 HRQOL subscales [9].

In order to better assess outcomes of patients with lymphedema, there is a need to incorporate the patient's perspective. Patient-reported outcome measures (PROMs) are questionnaires used to measure the patient's perspective of their own outcomes. The essence of a PROM is to quantify the impact of a disease or an intervention on a patient's everyday life [10]. Due to the increasing interest in patient involvement, PROMs are progressively used in research and clinical practice to better understand the impact of treatment on patients $[11,12]$.

Prior to the development of the LYMPH-Q Upper Extremity Module, a review by Cornelissen et al. concluded that the Lymph-ICF and LyQLI questionnaires are the two most sufficient questionnaires to determine the quality of life in patients with BCRL [13]. The review measured the questionnaires on the following topics: physical function, mental function, daily activities, hobbies and job, mobility, social activities, and sexual function. A Danish version of Lymph-ICF already exists [14]. However, a recent systematic review and COSMIN analysis on patient reported outcome measures in lymphedema by Beelen et al. concluded that the existing lymphedema-specific PROMs lack evidence of a sufficient development process and do not live up to the methodological quality standards [15]. Therefore, they recommended a new lymphedema-specific PROM that is based on extensive qualitative input from lymphedema patient which is adequately validated in studies showing good methodological quality to be developed.

The LYMPH-Q Upper Extremity Module was recently developed according to international guidelines and methods for PROM development [16-21]. The objective of this study was to perform a Danish translation and cultural adaption of the LYMPH-Q Upper Extremity Module.
Table 1 Overview of the LYMPH-Q Upper Extremity Module

\begin{tabular}{ll}
\hline Scale: & $\begin{array}{l}\text { Number of } \\
\text { questions: }\end{array}$ \\
\hline Symptoms & 15 \\
Function & 12 \\
Appearance & 10 \\
Distress & 12 \\
Information & 9 \\
Sleeve & 10 \\
Total: & $\mathbf{6 8}$
\end{tabular}

\section{Methods}

The LYMPH-Q Upper Extremity Module consists of six independently functioning scales: symptoms ( $n=15$ items), function ( $n=12$ items), appearance ( $n=10$ items), psychological ( $n=12$ items), information ( $n=9$ items), and sleeve $(n=10$ items) (Table 1). Translation and cultural adaption of the scales followed best-practice guidelines from the International Society for Pharmacoeconomics and Outcomes Research (ISPOR) [22] and the World Health Organization (WHO) [23] and involved the following steps:

1. Preparation: Approval for the study was sought from the Danish Data Protection Agency (journal number: 18/47762). According to Danish Law, questionnaires do not require approval from the National Committee on Health Research Ethics [24]. Permission was obtained from the developers of the LYMPH-Q Upper Extremity Module to translate the scales.

2. Forward translation: Two independent forward translations were performed by a professional translator and a plastic surgeon resident. Both translators had Danish as their mother tongue and were fluent in English. A reconciliation and harmonization meeting between the two forward translators to compare their translations and resolve any inconsistencies led to a harmonized Danish version 1.

3. Back translation: A professional translator, with English as their mother tongue and fluent in Danish, performed a back translation of the harmonized Danish version 1 into English. The back translation was compared to the original English version by the original developers of the LYMPH-Q Upper Extremity Module. Discrepancies in the meaning of any items, instructions, or response options were re-translated and evaluated until a satisfactory result was obtained. Changes made to the translation resulted in Danish version 2.

4. Expert panel: An expert panel meeting was held to determine if the Danish translation (version 2) was considered comprehensive and comprehensible and relevant 
by experts. According to the WHO guidelines, the expert panel should consist of bilingual clinicians with expertise in the given patient group as well as the translators. Reconciliation after the expert meeting led to the Danish version 3.

5. Cognitive debriefing interviews: Thorough cognitive interviews were conducted with patients from the target population. A representative sample of patients was sought that varied by parameters such as ISL (International Society of Lymphology) grade and time since lymphedema diagnosis. The purpose of these interviews was to determine if patients appropriately understood the translation of the items, instructions, and response options, if they are relevant, and if there is any missing content. Participants who expressed any difficulty understanding an item, instruction, or response option were asked to suggest changes to the translation. Compiled results from the patient interviews were reviewed and any items, instructions, or response options that were difficult were the re-translated and tested in a new sample. Results from the cognitive debriefing interviews led to the development of Danish version 4.

6. Proofreading: Danish version 4 was proofread by two clinicians to ensure there were no errors producing the final Danish version of the LYMPH-Q Upper Extremity Module.

\section{Results}

\section{Forward translation}

Translators were instructed to conduct a conceptual rather than literal translation. There was a high level of consensus between the two forward translations; however, individual differences between the translations for 10 items/instructions/response options were discussed due to variations in wording. For example, one translator used the Danish equivalent of "last week" where the other translator has used "past week." The number of synonyms used in the content of the distress scale represented a challenge. We found a lack of synonyms in Danish to adequately translate all the items. The number of synonyms is not as high in Danish as in English. A thorough discussion between the two translators aided a sufficient translation. The two translators reached consensus resulting in a harmonized Danish version 1 of the LYMPH-Q Upper Extremity Module.

\section{Back translation}

The back translation and the original English version were compared. This process was an interactive discussion between the translators and the LYMPH-Q Upper Extremity Module developers. Back translation review identified 14 items/instructions/response options that required re-translation. An example of an item that required re-translation was "Arm symptoms disturbing your sleep (e.g., pain, swelling)?." The Danish translation read as "Symptoms in your arm (e.g., pain, swelling) affect how you sleep at night?" After discussion with the LYMPH-Q Upper Extremity Module developers to better understand the meaning of the item, it was re-translated. The end result was a Danish translation that preserved the meaning of the original English version. This step resulted in a Danish version 2 of the LYMPH-Q Upper Extremity Module.

\section{Expert panel meeting}

The expert panel consisted of the primary investigator, the two professional bilingual translators (forward and back translators), a breast surgeon/plastic surgeon, physiotherapist specialized in lymphedema treatment, a medical doctor specialized in lymphedema research, and a medical doctor specialized in PROM research.

The experts identified 10 items/instructions/response options that required re-evaluation. For example, experts felt that the translation of the item "holding a bag of groceries?" was redundant because in Denmark there has previously been consensus about not using the lymphedema arm in order to relieve it. However, the newest treatment plans recommend use of the arm in a way that does not course pain or worsening of symptoms. Consequently, the question has some relevance in Denmark and was consequently included in the Danish version. This example illustrates the importance of the expert panel meeting, where clinicians in daily contact with the specific patient group can contribute with their experiences and the most updated clinical knowledge. The expert panel meeting provided important information that resulted in a Danish version 3 of the LYMPH-Q Upper Extremity Module.

\section{Cognitive debriefing interviews}

Interviews were conducted with 10 female patients with breast cancer related arm lymphedema. For patient characteristics, see Table 2. Participants did not identify any problems with understanding the items, instructions, or response options and no content was considered to be missed or irrelevant. No changes were made after cognitive debriefing interviews. 
Table 2 Patient characteristics

\begin{tabular}{ll}
\hline Sex: & Female: $n=10$ \\
& Male: $n=0$ \\
\hline Age (years): & Median: 63 (range: 41-75) \\
Years of education: & Median: 15 (range: $9-18$ ) \\
Grade of lymphedema (ILS): & Grade $0: n=0$ \\
& Grade $1: n=4$ \\
& Grade 2: $n=6$ \\
& Grade 3: $n=0$ \\
Time since breast cancer treatment (years): & Median: 6 \\
& Range: $2-10$ \\
Time since lymphedema diagnosis (years): & Median: 5 \\
& Range: 3-8 \\
Lymphedema treatment: & Arm sleeve: $n=2$ \\
& Manual drainage from physiotherapists: $n=1$ \\
& Arm sleeve and manual drainage from physi- \\
& otherapists: $n=7$
\end{tabular}

\section{Proofreading by two clinicians}

Two clinicians with experience with lymphedema patients and with translating PROMs proofread the Danish version 4. Minor grammatical changes were made and resulted in the development of the final Danish version of the LYMPH-Q Upper Extremity Module.

\section{Discussion}

To perform the translation and cultural adaption of the LYMPH-Q Upper Extremity Module, we used guidelines from ISPOR and WHO. By combining these two guidelines, we used a six-step approach that included forward and back translation, an expert panel meeting, and cognitive interviews. We found the expert panel meeting and cognitive interviews to be very helpful in creating a Danish version that was clinically relevant and understandable for BCRL patients.

The translation process and cultural adaption process led to the development of the Danish version of the LYMPH-Q Upper Extremity Module that was conceptually equivalent to the original English version.

When comparing the LYMPH-Q Upper Extremity Module to the Lymph-ICF, there are a few similarities and differences. First, both PROMs cover the same six out of seven topics that the review evaluated the questionnaires on (except sexual function). Second, both questionnaires can be used as individual scales for a more specific insight or to provide an overall score.

There are some benefits when using the LYMPH-Q Upper Extremity Module. First, it consists of 68 questions (see Table 2) which potentially creates a more detailed insight on the HR-QOL for BCRL patients compared to the 29 questions that make up Lymph-ICF.

Second, the method used to calculate the score in the LYMPH-Q Upper Extremity Module is developed according to Rasch Measurement Theory (RMT) where the revised Lymph-ICF was evaluated looking at intraclass correlation coefficients for test-retest reliability and Cronbach's alpha coefficients for internal consistency [25]. Hobart and Cano conclude that RMT has several advantages when measuring PRO in health care [26].

Third, the LYMPH-Q Upper Extremity Module includes questions concerning arm sleeve and information about lymphedema, which was found to be important through the qualitative interviews in the development phase [27]. Lymph ICF does not include these topics.

Lastly, we used advanced translation which is a process where a pre-final version of the PROM is translated from the original language into a foreign language (Danish). The purpose is to get feedback from the translation process including which items were problematic to translate [28]. This information is taken into account and used to improve and finalize the PROM via, e.g., item reduction. An example of this interactive development of the final PROM is from our cognitive interviews: A few patients highlighted the phrasing of an item in the information scale as problematic: "Which breast cancer patients are most likely to develop lymphedema?" They had difficulty understanding the question and they were uncertain if it was in terms of cancer (e.g., ductal carcinoma, lobular carcinoma, or estrogen receptor status) or the treatment type (e.g., operation, operation and chemotherapy, or operation, chemotherapy, and radiotherapy). In our feedback to the developers of the LYMPH-Q Upper Extremity Module, this problem was highlighted. Consequently, the item 
was dropped during item reduction and is not a part of the final version of the PROM.

Our study has a few limitations. First, the study was not performed as a multicenter study. The study population only included data from patients treated for BCRL in the Region of Southern Denmark at Odense University Hospital, Odense, Denmark. However, due to the small size of our country, we still find the data representable for the entire country. Furthermore, the Danish Breast Cancer Group (DCBG) has developed national guidelines that secure all patients receive the same standard treatment at all Danish hospitals [29]. Consequently, our patients are likely to experience the same challenges as all Danish BCRL patients. Second, we only interviewed female patients because no male patients were being treated for BCRL at our institution during the inclusion period. The majority of breast cancer patients are female (99\% in Denmark) but breast cancer can occur in male patients $(1 \%$ in Denmark) [1]. We believe that male patients experience many of the same symptoms as female patients, but this should be the focus of a future study.

In conclusion, an accurate translation and a proper cultural adaption are of great importance when implementing a PROM to another language or health care system. We found the translation methodology provided by ISPOR and WHO to be straightforward and easy to reproduce. The expert panel meeting and the cognitive interviews were useful steps taken to create a conceptually equivalent Danish version. The complete process led to a Danish translated and culturally adapted version of the LYMPH-Q Upper Extremity Module. The described method of translation and cultural adaption can be recommended for future translations of PROM's.

The final Danish version of the LYMPH-Q Upper Extremity Module will be available at http://qportfolio.org/.

Acknowledgements The first author would like to thank Marianne Holt, physiotherapist, and Camilla Bille, MD, for their contribution to the expert panel meeting.

Author contribution All authors contributed according to the journal guidelines.

Funding This work was funded by the Odense University Hospital Free Research Fund, Grant number 72-A3731.

Data availability Not relevant.

Code availability Not relevant.

\section{Declarations}

Ethics approval According to Danish Law, questionnaires do not require approval from the National Committee on Health Research Ethics.

Consent to participate All participants gave their consent.

Consent for publication All participants gave their consent.

Conflict of interest Christoffer Bing Madsen, Lotte Poulsen, Mads Gustaf Jørgensen, Mike Mikkelsen Lorenzen, Elena Tsangaris, Anne Klassen, and Jens Ahm Sørensen declare no competing interests.

Open Access This article is licensed under a Creative Commons Attribution 4.0 International License, which permits use, sharing, adaptation, distribution and reproduction in any medium or format, as long as you give appropriate credit to the original author(s) and the source, provide a link to the Creative Commons licence, and indicate if changes were made. The images or other third party material in this article are included in the article's Creative Commons licence, unless indicated otherwise in a credit line to the material. If material is not included in the article's Creative Commons licence and your intended use is not permitted by statutory regulation or exceeds the permitted use, you will need to obtain permission directly from the copyright holder. To view a copy of this licence, visit http://creativecommons.org/licenses/by/4.0/.

\section{References}

1. NORDCAN, Association of the Nordic Cancer Registries. 2019.

2. Grada AA, Phillips TJ (2017) Lymphedema: pathophysiology and clinical manifestations. J Am Acad Dermatol 77(6):1009-1020

3. DiSipio T, Rye S, Newman B, Hayes S (2013) Incidence of unilateral arm lymphoedema after breast cancer: a systematic review and meta-analysis. Lancet Oncol 14(6):500-515

4. Chachaj A, Malyszczak K, Pyszel K, Lukas J, Tarkowski R, Pudelko M et al (2010) Physical and psychological impairments of women with upper limb lymphedema following breast cancer treatment. Psychooncology 19(3):299-305

5. Asdourian MS, Skolny MN, Brunelle C, Seward CE, Salama L, Taghian AG (2016) Precautions for breast cancer-related lymphoedema: risk from air travel, ipsilateral arm blood pressure measurements, skin puncture, extreme temperatures, and cellulitis. Lancet Oncol 17(9):e392-405

6. Hidding JT, Viehoff PB, Beurskens $\mathrm{CH}$, van Laarhoven HW, Nijhuis-van der Sanden MW, van der Wees PJ (2016) Measurement properties of instruments for measuring of lymphedema: systematic review. Phys Ther 96(12):1965-81

7. Terada M, Yoshimura A, Sawaki M, Hattori M, Naomi G, Kotani $\mathrm{H}$ et al (2020) Patient-reported outcomes and objective assessments with arm measurement and bioimpedance analysis for lymphedema among breast cancer survivors. Breast Cancer Res Treat 179(1):91-100

8. Sackey H, Johansson H, Sandelin K, Liljegren G, MacLean G, Frisell J et al (2015) Self-perceived, but not objective lymphoedema is associated with decreased long-term health-related quality of life after breast cancer surgery. Eur J Surg Oncol 41(4):577-584

9. Jørgensen MG, Toyserkani, N., Hansen, F., Bygum, A., Sørensen, J.A. . The impact of lymphedema on health-related quality of life up to 10 years after breast cancer treatment npj Breast Cancer. 2021. 
10. Cano SJ, Klassen A, Pusic AL (2009) The science behind qualityof-life measurement: a primer for plastic surgeons. Plast Reconstr Surg 123(3):98e-106e

11. Sharma K, Steele K, Birks M, Jones G, Miller G (2019) Patientreported outcome measures in plastic surgery: an introduction and review of clinical applications. Ann Plast Surg 83(3):247-252

12. Wormald JCR, Rodrigues JN (2018) Outcome measurement in plastic surgery. J Plast Reconstr Aesthet Surg JPRAS 71(3):283-289

13. Cornelissen AJM, Kool M, Keuter XHA, Heuts EM, Piatkowski de Grzymala AA, van der Hulst R et al (2018) Quality of life questionnaires in breast cancer-related lymphedema patients: review of the literature. Lymphat Res Biol 16(2):134-9

14. Grarup KR, Devoogdt N, Strand LI (2019) The Danish version of Lymphoedema Functioning, Disability and Health Questionnaire (Lymph-ICF) for breast cancer survivors: translation and cultural adaptation followed by validity and reliability testing. Physiother Theory Pract 35(4):327-340

15. Beelen LM, van Dishoeck AM, Tsangaris E, Coriddi M, Dayan $\mathrm{JH}$, Pusic AL, et al. Patient-reported outcome measures in lymphedema: a systematic review and COSMIN analysis. Ann Surg Oncol. 2020.

16. Lasch KE, Marquis P, Vigneux M, Abetz L, Arnould B, Bayliss $M$ et al (2010) PRO development: rigorous qualitative research as the crucial foundation. Qual Life Res 19(8):1087-1096

17. Patrick DL, Burke LB, Gwaltney CJ, Leidy NK, Martin ML, Molsen E et al (2011) Content validity-establishing and reporting the evidence in newly developed patient-reported outcomes (PRO) instruments for medical product evaluation: ISPOR PRO Good Research Practices Task Force report: part 2-assessing respondent understanding. Value Health 14(8):978-988

18. Aaronson N, Alonso J, Burnam A, Lohr KN, Patrick DL, Perrin E et al (2002) Assessing health status and quality-of-life instruments: attributes and review criteria. Qual Life Res 11(3):193-205

19. Mokkink LB, Terwee, C. B., Patrick, D. L., Alonso, J., Stratford, P. W., Knol, D. L. et al. Cosmin (Consensus-based Standards for the selection of health Measurement Instruments) checklist 2009 [Available from: http://www.cosmin.nl. .

20. Mokkink LB, Terwee CB, Patrick DL, Alonso J, Stratford PW, Knol DL et al (2010) The COSMIN study reached international consensus on taxonomy, terminology, and definitions of measurement properties for health-related patient-reported outcomes. J Clin Epidemiol 63(7):737-745

21. Mokkink LB, Terwee CB, Patrick DL, Alonso J, Stratford PW, Knol DL et al (2010) The COSMIN checklist for assessing the methodological quality of studies on measurement properties of health status measurement instruments: an international Delphi study. Qual Life Res 19(4):539-549

22. Wild D, Grove A, Martin M, Eremenco S, McElroy S, VerjeeLorenz A et al (2005) Principles of good practice for the translation and cultural adaptation process for patient-reported outcomes (PRO) measures: report of the ISPOR task force for translation and cultural adaptation. Value Health 8(2):94-104

23. World Health Organization. Process of translation and adaption of instruments. [Available from: http://www.who.int/substance abuse/research_tools/translation/en/.

24. Act on research ethics review of health research projects, (2018).

25. De Vrieze T, Vos L, Gebruers N, De Groef A, Dams L, Van der Gucht E et al (2019) Revision of the lymphedema functioning, disability and health questionnaire for upper limb lymphedema (Lymph-ICF-UL): reliability and validity. Lymphat Res Biol 17(3):347-355

26. Hobart J, Cano S. Improving the evaluation of therapeutic interventions in multiple sclerosis: the role of new psychometric methods. Health Technol Assess. 2009;13(12):iii, ix-x, 1-177.

27. Klassen AF TE, Kaur MN, Poulsen L, Beelen LM, Jacobsen AM, Jørgensen MG, Sørensen JA, Vasilic D, Dayan J, Mehrara B, Pusic AL. Development and psychometric validation of a patient-reported outcome measure for arm lymphedema: LYMPHQ Upper Extremity Module. Ann Surg Oncol. 2021.

28. Acquadro C, Patrick DL, Eremenco S, Martin ML, Kulis D, Correia $\mathrm{H}$ et al (2017) Emerging good practices for Translatability Assessment (TA) of patient-reported outcome (PRO) measures. J Patient Rep Outcomes 2(1):8

29. DBCG. Danish Breast Cancer Group 2020 [Available from: https://www.dbcg.dk/.

Publisher's Note Springer Nature remains neutral with regard to jurisdictional claims in published maps and institutional affiliations. 